\title{
Ventral Prostate Gland
}

National Cancer Institute

\section{Source}

National Cancer Institute. Ventral Prostate Gland. NCI Thesaurus. Code C77623.

The ventral lobe of the prostate gland in animals. 